\title{
NONLINEAR TM WAVES GUIDED ON A PLANAR INTERFACE
}

\author{
M. WABIA ${ }^{a}$ AND G. ŻEGLIŃSKI ${ }^{b}$ \\ ${ }^{a}$ Institute of Physics, Technical University of Szczecin \\ Al. Piastów 48, 70-310 Szczecin, Poland \\ ${ }^{b}$ Department of Optical Telecommunication and Photonics \\ Technical University of Szczecin, 26 Kwietnia 10, 71-062 Szczecin, Poland
}

We study theoretically TM-polarized electromagnetic waves at a plane interface between two nonabsorbing, optically defocusing, nonlinear Kerr-type media. Numerical results are obtained for the dispersion equation and propagation constants of the field modes. The power guided by TM waves on interface was calculated and illustrated as a function of electric field value on interface.

PACS numbers: 42.65.-k

\section{Introduction}

The analysis of nonlinear TM guided waves is complicated by the inherent structure of the fields that contain two electric field components $E_{x}(x)$ and $E_{z}(x)$ which are $\pi / 2$ out of phase with one another [1]. This is why classical surface waves of the TM type on the nonlinear interface were only analysed by approximate methods using various assumptions [1-8].

In the paper [9] an analysis of electromagnetic boundary problem for TE field on a plane interface of two nonlinear media was presented. It was shown that apart from a surface wave, known from the literature, the boundary problem allows also for existence of the waves of other types. Nonlinear interface can guide also electromagnetic wave with the amplitude decaying in one region $(x>0)$ and oscillating in the second region $(x<0)$, which is non specific for classical surface wave. It is a new kind of wave and a question is if such wave with TM polarization can exist and be guided on the interface. In this work we shall try to find an answer for that question. 


\section{Geometry of the investigated problem}

Two nonlinear media of the Kerr type $(x>0$ and $x<0)$ divided by a plane interface and described with the following tensor of dielectric permittivity are considered:

$$
\widehat{\varepsilon}^{j}=\left(\begin{array}{ccc}
j_{\varepsilon_{11}} & 0 & 0 \\
0 & j_{\varepsilon} & 0 \\
0 & 0 & j_{\varepsilon_{33}}
\end{array}\right),
$$

where

$$
\begin{aligned}
& j_{\varepsilon_{11}}=j_{\varepsilon_{22}}=j_{\varepsilon}, \\
& j_{\varepsilon_{33}}=j_{\varepsilon_{1}}+\alpha_{j}\left|E_{z j}^{2}\right| .
\end{aligned}
$$

The index $j$ is the index of a medium and takes on the values $j=1,2$.

Nonlinearity of the Kerr type is enclosed in the component $\varepsilon_{33}$ of the tensor describing a medium. Values $j_{\mathcal{E}},{ }^{j} \varepsilon_{1}$ characterise linear permittivities and $\alpha_{j}$ is a coefficient of nonlinearity of a medium. Therefore, the medium $1(x>0)$ is described by the parameters ${ }^{1} \varepsilon,{ }^{1} \varepsilon_{1}, \alpha_{1}$, the medium $2(x<0)$ is described by the parameters ${ }^{2} \varepsilon,{ }^{2} \varepsilon_{1}, \alpha_{2}$, and $E_{z}$ is the longitude component of the electromagnetic field. The geometry of the problem is illustrated in Fig. 1.

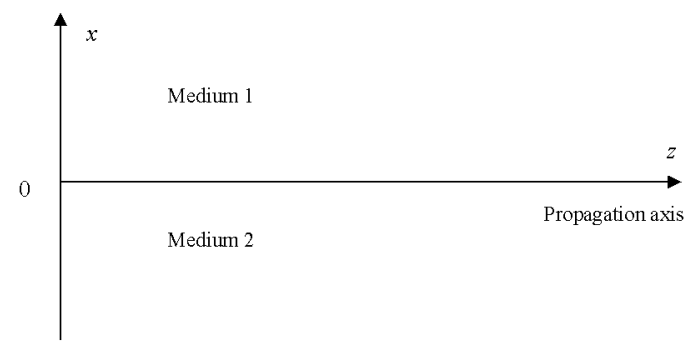

Fig. 1. Geometry of considered problem for TM waves.

\section{Maxwell equations for considered regions}

Maxwell equations describing TM field in media 1 and 2 take on the general form

$$
\begin{aligned}
& \frac{\partial H_{y}}{\partial z}=\mathrm{i} \omega \varepsilon_{0} \varepsilon_{11} E_{x}, \\
& \frac{\partial H_{y}}{\partial x}=-\mathrm{i} \omega \varepsilon_{0} \varepsilon_{33} E_{z}, \\
& \frac{\partial E_{x}}{\partial z}-\frac{\partial E_{z}}{\partial x}=-\mathrm{i} \omega \mu_{0} H_{y},
\end{aligned}
$$

where $\varepsilon_{0}$ and $\mu_{0}$ - the permittivity and permeability of free space, respectively. TM field is completely described by three components $E_{x}, E_{z}$, and $H_{y}$. 


\section{Wave equation for TM field in considered region}

We assume that the considered electromagnetic field is slowly variable and it does not depend on coordinate $y$. In such a case the component $E_{z j}(x, z, t)$ can take on the form

$$
E_{z j}(x, z, t)=E_{z j}(x) \exp \left[\mathrm{i}\left(k_{0} h z-\omega t\right)\right]
$$

where $h$ is propagation constant of a mode, $k_{0}$ is the free space wave number. The other components of the field, $E_{x}, H_{y}$, are defined univocally by the Maxwell equations (4)-(6). Using (2), (3), and (7) and transforming Maxwell equations, we obtain the wave equation for amplitude $E_{z j}(x)$ in $j$ region

$$
\frac{\mathrm{d}^{2} E_{j}}{\mathrm{~d} x^{2}}-\frac{k_{j}^{2}}{j_{\varepsilon}}\left({ }^{j_{\varepsilon}} \varepsilon_{1}+\alpha_{j}\left|E_{j}\right|^{2}\right) E_{j}=0
$$

where

$$
\begin{aligned}
& E_{j} \equiv E_{z j}(x), \\
& k_{j}=k_{0}\left(h^{2}-j_{\varepsilon}\right)^{1 / 2} .
\end{aligned}
$$

\section{Solution of wave equation}

The first integral of wave equation (8) takes on the form

$$
\left(\frac{\mathrm{d} E_{j}}{\mathrm{~d} x}\right)^{2}-\frac{k_{j}^{2}}{\varepsilon^{j}}\left(j_{\varepsilon_{1}} E_{j}^{2}+\frac{\alpha_{j}}{2} E_{j}^{4}\right)=C_{j} .
$$

Properties and the form of the last solution are determined by the values $k_{j}^{2}, \alpha_{j}$, and $C_{j}$. In the case when $k_{j}^{2}>0, \alpha_{j}>0$ (focusing medium), $C_{j}>0$, then a solution of wave equation (8) is expressed by the Jacobi cs function

$$
E_{j}(x)=\Omega_{j} \operatorname{cs}\left[\sigma_{j}\left(x-x_{0 j}\right) \mid m_{j}\right],
$$

where

$$
\begin{aligned}
& \operatorname{cs}(u \mid m)=\operatorname{cn}(u \mid m) / \operatorname{sn}(u \mid m), \\
& \Omega_{j}=\left[\frac{2 C_{j}}{a_{j}-\left(a_{j}^{2}-4 b_{j} C_{j}\right)^{1 / 2}}\right]^{1 / 2}, \\
& \sigma_{j}=\left[\frac{a_{j}+\left(a_{j}^{2}-4 b_{j} C_{j}\right)^{1 / 2}}{2}\right]^{1 / 2}, \\
& m_{j}=\frac{2\left(a_{j}^{2}-4 b_{j} C_{j}\right)^{1 / 2}}{\left[a_{j}+\left(a_{j}^{2}-4 b_{j} C_{j}\right)^{1 / 2}\right]} .
\end{aligned}
$$

The parameters $a_{j}$ and $b_{j}$ are expressed by the formulas

$$
a_{j}=\frac{k_{j}^{2} \xi_{\varepsilon_{1}}}{\varepsilon_{j}},
$$




$$
b_{j}=\frac{\alpha_{j} k_{j}^{2}}{2 \varepsilon_{j}},
$$

and $x_{0 j}$ is an integral constant of Eq. (11).

From the form of the solution of Eq. (12), it could be seen that the electric field can have, in this case, a singularity in the point $x=x_{0 j}$ and such solution has to be rejected form the physical point of view.

In the case when $k_{j}^{2}>0, \alpha_{j}<0$ (defocusing medium), $C_{j}>0$, then the solution of wave equation is expressed by the Jacobi cosine

$$
E_{j}(x)=\delta_{j} \operatorname{cn}\left[\gamma_{j}\left(x-x_{0 j}\right) \mid m_{j}\right]
$$

where

$$
\begin{aligned}
& \delta_{j}=\left[\frac{a_{j}+\left(a_{j}^{2}+4\left|b_{j}\right| C_{j}\right)^{1 / 2}}{2\left|b_{j}\right|}\right]^{1 / 2}, \\
& \gamma_{j}=\left(a_{j}^{2}+4\left|b_{j}\right| C_{j}\right)^{1 / 4}, \\
& m_{j}=\frac{a_{j}+\left(a_{j}^{2}+4\left|b_{j}\right| C_{j}\right)^{1 / 2}}{2\left(a_{j}^{2}+4\left|b_{j}\right| C_{j}\right)^{1 / 2}},
\end{aligned}
$$

and the expressions $a_{j}$ and $b_{j}$ are given further by (17) and (18), respectively. Jacobi function $\operatorname{cn}(u \mid m)$ has a period $4 K(M)$, where $K\left(m_{j}\right)=\int_{0}^{\pi / 2}\left(1-m_{j} \sin ^{2} \theta\right)^{-1 / 2} \mathrm{~d} \theta$ is the elliptic integral of the first type. From (19) one can calculate that $E_{j}(x)$ has its maxima in points $x_{n j}=x_{0 j}+n l_{j}, n=0,1,2, \ldots$ where the distances between maxima are $l_{j}=2 K\left(m_{j}\right) / \gamma_{j}$. Hence the electric field described by the expression (19) is periodical function of $x$.

In the case when $C_{j}=0$, then $m_{j}=1$ and we have $\operatorname{cn}(u \mid 1)=(\operatorname{ch} u)^{-1}$. We obtain then the no periodical and vanishing solution of the wave equation (8) in the form

$$
E_{j}=\left(\frac{a_{j}}{b_{j}}\right)\left\{\operatorname{ch}\left[a_{j}\left(x-x_{0}\right)\right]\right\}^{-1} .
$$

The electric field described by the solutions (19) or (23) does not have the singularity and these solutions can be used while defining a boundary problem on interface.

\section{Idea of boundary problem on nonlinear interface for TM field}

The above considerations of the solutions of nonlinear wave equation (8) showed that for defining the boundary problem for TM field on interface, we could use only nonsingular solution (19)-(23). This means that electromagnetic boundary problem for TM field in lossless nonlinear media is physically possible only if both media are of the defocusing type, i.e. when $\alpha_{1}<0, \alpha_{2}<0$.

Pure surface modes, which are not periodical and not vanishing for $x>0$ and $x<0$, are well known solution of a boundary problem [1-8]. Seeking for new 
solutions of the problem, we want to show the existence of such TM field modes, which are oscillating and periodical in one medium, vanishing in a second medium and fulfil the boundary condition on interface and in infinity.

While assuming a vanishing character of amplitude of a field in region 1 and its oscillating character in region 2, we also presume certain form for integral constants of Eq. (11), according to our previous considerations, namely

$$
\begin{aligned}
& C_{1}=0 \text { for } x>0, \\
& C_{2}>0 \text { for } x<0 .
\end{aligned}
$$

Then the solutions of nonlinear equation (8) take on the form (23) and (19).

Region $1, x>0$ and $\alpha_{1}<0$

$$
E_{1}=\left(\frac{a_{1}}{b_{1}}\right)\left\{\operatorname{ch}\left[a_{1}\left(x-x_{01}\right)\right]\right\}^{-1} \text {. }
$$

Region 2, $x<0$ and $a_{2}<0$

$$
E_{2}(x)=\delta_{2} \operatorname{cn}\left[\gamma_{2}\left(x-x_{02}\right) \mid m_{2}\right] \text {, }
$$

where $a_{1}, b_{1}, \delta_{2}, \gamma_{2}, m_{2}$ are given by the expressions (17), (18), (20), (21), and (22), respectively and $x_{01}$ and $x_{02}$ are the new integral constants and factors defining maxima of the functions (26) and (27).

\section{Conditions of continuity and integral constant $C_{2}$}

The boundary conditions for TM interface require the continuity of components $E_{z}$ and $H_{y}$ for $x=0$

$$
\begin{aligned}
& E_{1}(0)=E_{2}(0), \\
& H_{1}(0)=H_{2}(0),
\end{aligned}
$$

where $E_{j} \equiv E_{z j}, H_{j} \equiv H_{y j}$. From Maxwell equations (4) and (6) we have

$$
H_{j}(x)=\frac{i \omega \varepsilon_{0}{ }_{\varepsilon} \varepsilon}{k_{j}^{2}} \frac{\mathrm{d} E_{j}(x)}{\mathrm{d} x} .
$$

The relation (29) could be written in a form

$$
\left.\frac{\varepsilon_{j}}{k_{1}^{2}} \frac{\mathrm{d} E_{1}(x)}{\mathrm{d} x}\right|_{x=0}=\left.\frac{\varepsilon_{2}}{k_{2}^{2}} \frac{\mathrm{d} E_{2}(x)}{\mathrm{d} x}\right|_{x=0} .
$$

Using the explicit forms of components $E_{1}(x)$ and $E_{2}(x)$, the condition (31) and Eq. (11) allow for defining an integral constant $C_{2}$

$$
C_{2}=\frac{k_{2}^{2}}{{ }^{2}}\left[\left(\frac{{ }^{1} \varepsilon k_{2}^{2}}{{ }^{2} \varepsilon k_{1}^{2}} \varepsilon_{1}-{ }^{2} \varepsilon_{2}\right) E_{0}^{2}+\frac{1}{2}\left(\frac{{ }^{1} \varepsilon k_{2}^{2}}{{ }^{2} \varepsilon k_{1}^{2}} \alpha_{1}-\alpha_{2}\right) E_{0}^{4}\right],
$$

where $E_{0}$ is the value of electric field on interface $x=0$. 


\section{Dispersion equation and its discussion}

The conditions of continuity (28) and (29) of components of the field on interface $x=0$ give us the following relations:

$$
\begin{aligned}
& \delta \operatorname{cn}\left(\gamma^{2} x_{0}\right)=\left(\frac{a_{1}}{\left|b_{1}\right|}\right)^{1 / 2}\left[\operatorname{ch}\left(a_{1}^{1} x_{0}\right)\right]^{-1}, \\
& \frac{{ }^{2} \varepsilon}{k_{2}^{2}} \delta \gamma \operatorname{sn}\left(\gamma^{2} x_{0}\right) \operatorname{dn}\left(\gamma^{2} x_{0}\right)=\frac{{ }^{1} \varepsilon}{{ }^{1} k^{2}} \frac{a_{1}^{3 / 2}}{\left|b_{1}\right|^{1 / 2}} \operatorname{th}\left(a_{1}^{1} x_{0}\right)\left[\operatorname{ch}\left(a_{1}^{1} x_{0}\right)\right]^{-1} .
\end{aligned}
$$

Replacing (33) with (34) we obtain the dispersion equation, which determines the TM field modes on interface

$$
{ }^{1} \varepsilon a_{1} k_{2}^{2} \operatorname{th}\left(a_{1} x_{01}\right) \operatorname{cn}\left(\gamma_{2} x_{02}\right)={ }^{2} \varepsilon k_{1}^{2} \operatorname{sn}\left(\gamma_{2} x_{02}\right) \operatorname{dn}\left(\gamma_{2} x_{02}\right) .
$$

The last equation can be easily reduced on explicit form of an equation, determining the propagation constants $h$ of these modes. Assuming a certain value of the electric field $E_{0}$ on the interface, we have

$$
E_{0}=\left(\frac{a_{1}}{b_{1}}\right)^{1 / 2}\left[\operatorname{ch}\left(a_{1} x_{01}\right)\right]^{-1},
$$

and

$$
E_{0}=\delta_{2} \operatorname{cn}\left(\gamma_{2} x_{02} \mid m_{2}\right)
$$

Using the last relations, the dispersion equation (35) could be transformed into the form, in which the value of $E_{0}$ field and the coefficients of nonlinearity $\alpha_{1}$ and $\alpha_{2}$ exist in various powers. As the coefficients of nonlinearity $\alpha_{1}$ and $\alpha_{2}$ are of the order of $10^{-12} \mathrm{~m}^{2} / \mathrm{V}^{2}$, so the expressions containing $\alpha_{1}$ and $\alpha_{2}$ in the powers $n \geq 2$ cannot be avoided because of their infinitesimal contribution. Easy transformations lead us then towards the dispersion equation with its form

$$
\left(h^{2}-{ }^{2} \varepsilon\right)\left(2^{2} \varepsilon+\frac{{ }^{1} \varepsilon}{{ }^{2} \varepsilon} \alpha_{1}\right)+\alpha_{2}\left(h^{2}-{ }^{1} \varepsilon\right)=0 .
$$

The roots of the last equations are easy to determine and they have the form

$$
h= \pm \sqrt{\frac{2 \varepsilon_{2}^{2}+2 \varepsilon_{1} \alpha_{1}}{2 \varepsilon_{2}+\frac{\varepsilon_{1}}{\varepsilon_{2}} \alpha_{1}+\alpha_{2}}} \approx \sqrt{\varepsilon_{2}} .
$$

A propagation constant $h$, as one can see, is determined by the parameters of a boundary media and does not depend on the value of $E_{0}$ field on interface. This means that in considered structure, where the parameters of media are given, we can guide only one mode of TM electric field with the propagation constant (39).

\section{Power guided by TM field on the interface}

The power flux in optional region is determined by the average value of the Poynting vector, which is given for TM field in considered structure as

$$
\langle\boldsymbol{S}\rangle=\frac{1}{2} \operatorname{Re}\left[-i\left(E_{z} H_{y}^{*}\right)+k\left(E_{x} H_{y}^{*}\right)\right] .
$$


Using Maxwell equations for TM field (4)-(6), a component of the flux on the interface in $j$ region could be expressed in the form

$$
\langle\mathrm{S}\rangle_{\mathrm{z}}=\frac{1}{2} \operatorname{Re}\left[\frac{\omega \varepsilon_{0}{ }^{j_{\varepsilon}} k_{0} h}{k_{j}^{4}} \frac{\partial E_{z}}{\partial x} \frac{\partial E_{z}^{*}}{\partial x}\right],
$$

and the power guided in that direction is defined by integration of the last expression.

\subsection{Power guided in region $1(x>0)$}

Using (41) and (26) we obtain

$$
P_{1}=\frac{\omega \varepsilon_{0}^{1} \varepsilon k_{0} h}{2 k_{1}^{4}} \int_{0}^{\infty} \frac{\partial E_{z}}{\partial x} \frac{\partial E_{z}^{*}}{\partial x} \mathrm{~d} x=\frac{\omega \varepsilon_{0}^{1} \varepsilon k_{0} h}{6 k_{1}^{4} b_{1}}\left[1+\operatorname{th}^{3}\left(a_{1} x_{01}\right)\right],
$$

where $k_{1}, a_{1}, b_{1}$ are determined by the relations (10), (17), and (18), respectively.

The dependence of power $P_{1}$ on the value of electric field $E_{0}$ strength on the interface is illustrated in Fig. 2.

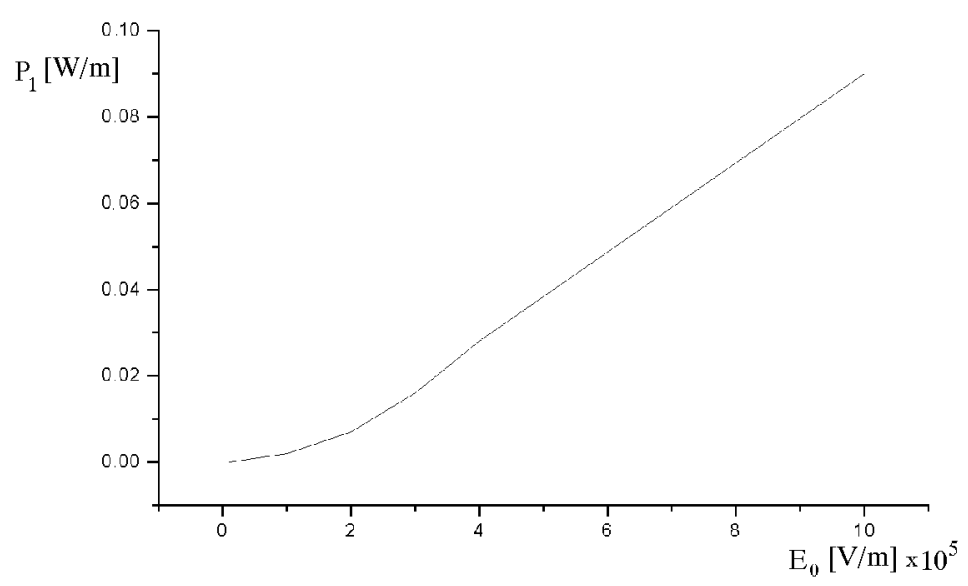

Fig. 2. Power guided on the interface in region 1 as a function of $E_{0}$ field.

$$
\text { 9.2. Power guided in region } 2(x<0)
$$

According to (4) and (27) the power guided in this region can be expressed in the form

$$
\begin{aligned}
P_{2}= & \frac{\omega \varepsilon_{0}^{2} \varepsilon k_{0} h}{2 k_{2}^{4}} \int_{-\infty}^{0} \frac{\partial E_{z}}{\partial x} \frac{\partial E_{z}^{*}}{\partial x} \mathrm{~d} x \\
& =\frac{\omega \varepsilon_{0}^{2} \varepsilon k_{0} h}{4 k_{2}^{4}}\left\{m_{2}^{-2}\left[-E\left[a m(0), m_{2}\right]+E\left[\operatorname{am}\left(2 K\left(m_{2}\right)\right), m_{2}\right]\right]\right. \\
& \left.-m_{2}^{2} \gamma_{2} \in t_{-\infty}^{0} \operatorname{sn}^{4}\left[\gamma_{2}\left(x-x_{02}\right)\right] \mathrm{d} x\right\}
\end{aligned}
$$


where $E$ and $K$ are the respective elliptic integrals. The power $P_{2}$ can be further analysed numerically in an easy way. The dependence of power $P_{2}$ on the value of electric field $E_{0}$ strength on the interface is illustrated in Fig. 3. Calculations of the powers $P_{1}$ and $P_{2}$ were made for the case when a medium 1 is characterised by the coefficients: $\varepsilon_{\varepsilon_{1}}=1.55, \alpha_{1}=6.37 \times 10^{-11} \mathrm{~m}^{2} / \mathrm{V}^{2}$ and a medium 2 by coefficients: ${ }^{2} \varepsilon_{2}=1.83, \alpha_{1}=8.88 \times 10^{-10} \mathrm{~m}^{2} / \mathrm{V}^{2}[4,5]$. The wave of the TM mode is of the length $\lambda=0.633 \mu \mathrm{m}$.

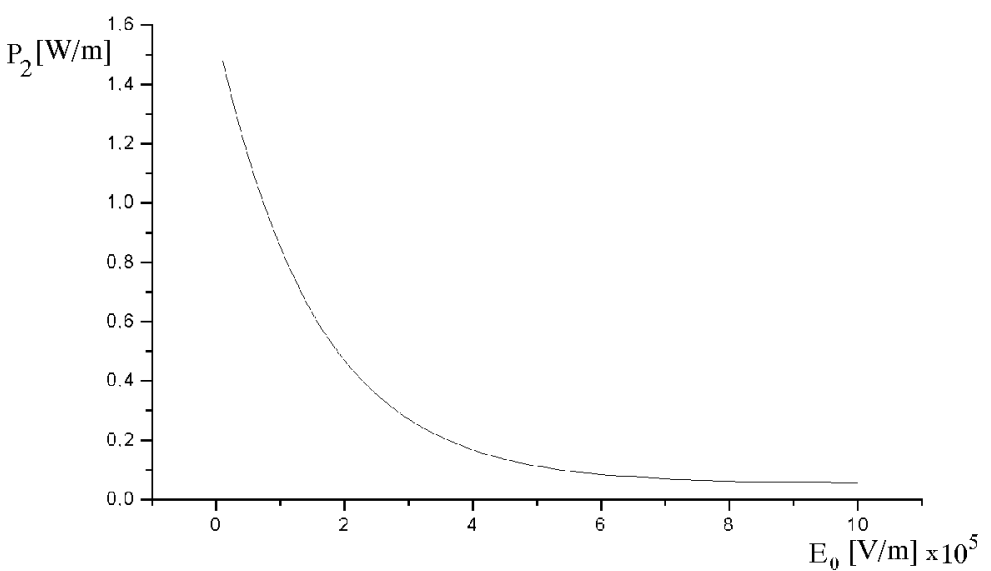

Fig. 3. Power guided on the interface in region 2 as a function of $E_{0}$ field.

\section{Discussion of obtained results}

a) Electromagnetic field with polarisation TM and variable amplitude perpendicular to interface can be excited on nonlinear interface between two nonlinear media of the Kerr type, which are described by uniaxial tensor (1) and in approximation (2), (3).

b) It means that for $x>0$ amplitude quickly decays in the direction perpendicular to interface, and for $x<0$ amplitude oscillates with a certain period of oscillation.

c) The wave can be however excited only in the case when both adjacent media are characterised by negative nonlinear coefficients $\alpha_{j}<0$ (defocusing media).

d) For the chosen nonlinear approximation (2), (3) the constants of propagation of TM field on interface are only weakly dependent on the value of guided power and thus we assume that no such dependence exists.

e) A real part of Poynting vector, perpendicular to interface is equal to zero. In spite of the amplitude oscillation the wave does not propagate in that direction. Only oscillation of the power proceeds. The half of the period of 
the Poynting vector is directed towards positive values of $x$, the second half is directed towards the negative values. The time average value of oscillation of the Poynting vector perpendicular to interface is equal to zero.

f) Such energetic behaviour is characteristic of surface waves. Typical surface wave has however the amplitude vanishing on the both sides of interface.

g) TM wave described in that paper can be called the wave of the surface type or quasi-surface wave because of the amplitude behaviour which is different than that of the typical surface wave.

h) A wave with similar characteristics appears in complete internal reflection on the nonlinear interfaces.

i) TM wave guided on nonlinear interface, being discussed in that paper, is a new kind of wave, excited on that interface.

\section{References}

[1] V.M. Agranowich, V.S. Babichenko, V.Ya. Chernyak, Sov. Phys. JETP Lett. 32, 512 (1981).

[2] N. Akhmediev, Zh. Eksp. Teor. Fiz. 84, 1907 (1983).

[3] T. Seaton, J.D. Valera, B. Svenson, G.I. Stegeman, Opt. Lett. 10, 149 (1985).

[4] D. Michalache, G.I. Stegeman, G.T. Seaton, E.M. Wring, R. Zanoni, A.D. Boardman, T. Twardowski, Opt. Lett. 12, 187 (1987).

[5] A.D. Boardman, A.A. Maradudin, G.I. Stegeman, T. Twardowski, E.M. Wright, Phys. Rev. A 35, 1159 (1987).

[6] A.D. Boardman, P.E. Egan, F. Lederer, U. Langbein, D. Michalake, in: Nonlinear Surface Electromagnetic Phenomena, Eds. H.E. Pnath, G.I. Stegeman, North-Holland, Amsterdam 1991, Ch. 2.

[7] A.D. Boardman, P.E. Egan, T. Twardowski, M. Wilkins, in: Nonlinear Waves in Solid State Physics, Ed. A.D. Boardman, NATO Advanced Study Institute, Vol. 247, Series B: Physics, Plenum Press, New York 1992, Ch. 1.

[8] E. Wright, G. Stegeman, in: Anisotropic and Nonlinear Optical Waveguides, Eds. C.G. Someda, G. Stegeman, Elsevier Sci. Publ., New York 1991, p. 117.

[9] M. Wabia, G. Żegliński, Acta Phys. Pol. A 99, 87 (2001). 